\title{
Idiopathic pulmonary fibrosis management: caring is sparing
}

\author{
Stefano Nava ${ }^{1,2}$, Irene Prediletto ${ }^{2}$ \\ ${ }^{1}$ Department of Clinical, Integrated and Experimental Medicine (DIMES), Alma Mater Studiorum University of Bologna, Bologna, Italy; \\ ${ }^{2}$ Respiratory and Critical Care Unit, Sant'Orsola-Malpighi Hospital, Bologna, Italy \\ Correspondence to: Irene Prediletto, MD, PhD. Respiratory and Critical Care Unit, Sant'Orsola-Malpighi Hospital, Buliding \# 15, Via Massarenti 9, \\ 40138 Bologna, Italy. Email: irene.prediletto@aosp.bo.it. \\ Comment on: Hambly N, Goodwin S, Aziz-Ur-Rehman A, et al. A cross-sectional evaluation of the idiopathic pulmonary fibrosis patient satisfaction \\ and quality of life with a care coordinator. J Thorac Dis 2019;11:5547-56.
}

Submitted Mar 27, 2020. Accepted for publication Apr 07, 2020.

doi: $10.21037 /$ jtd.2020.04.44

View this article at: http://dx.doi.org/10.21037/jtd.2020.04.44

Idiopathic pulmonary fibrosis (IPF) is a chronic, progressive and scarring lung disease characterized by irreversible decline in lung function leading to chronic respiratory failure (1). About 5 million of people are affected globally (2) and average life expectancy following diagnosis is about 4 years. Health Related Quality of life (HRQoL) is low and characterized by symptoms such as shortness of breath, dry cough and limited exercise tolerance $(3,4)$. New effective therapies were approved for IPF in the last decade; nintedanib and pirfenidone showed benefits in term of progression of the disease slowing the decline of forced vital capacity (FVC) and reducing the number of acute exacerbations. No randomized clinical trial demonstrates a definite reduction of mortality rate so far, so that the prognosis of the disease remains poor (5-7). A recent Medline search by these Authors (January 2020), have found around 5,500 original articles, meta-analysis or reviews on the topic "IPF and treatment", the very large majority of the studies being focused on the pharmacological treatments and only few dozen on the "integrated care" of these patients. This is rather surprising, since when up-todate no drug seems to impact the life expectancy of these patients; emphasis should be given to the quality of life of the patients and implementing symptom management and patient's disease perception (8).

Indeed, it has been recently shown that despite the overall tolerance to the treatments is acceptable and the dropout rate is low, a relatively high number of patients refuse treatments. Some studies explored this issue and it was found that patients are scared by the possible side effects or were not fully convinced of the efficacy of the drugs; in some cases, the family doctor suggested patients not to receive IPF drug $(9,10)$. Overall, this highlights the need of dedicated comprehensive and "holistic" programs for IPF patients, since the early stage of the diagnosis.

Health care system models are not designed to fully satisfy patient-centered care especially for chronic diseases such as progressive interstitial lung disease, unfortunately. In an attempt to fill this gap, in this issue of the journal Hambly and coworkers (11) investigated in a cross sectional study the effect of a dedicated care coordinator on IPF patient satisfaction and HRQoL. In this study forty patients were enrolled divided equally in a group followed by a physician with high coordinator use (HCU) and a group followed by a physician with low coordinator use (LCU). In the HCU clinic, the coordinator was involved with patient management, education, treatment, research and administration. In the LCU clinic the coordinator only assisted with treatment related tasks.

Being the figure of care coordinator prone to differences depending on the geographical location and the system of care around the world, the Authors meant with this professional a dedicated person "is similar to a specialist IPF nurse with additional responsibilities including indirect (e.g., drug reimbursement applications) and palliative care, and research coordination". The inclusion of a care coordinator figure in the management of IPF patients lead to improved patient satisfaction in this preliminary study. Moreover, to transfer some part of the care from the threating physician to the coordinator was found to spare physician time without reducing patient HRQoL (11).

Important considerations could be made concerning the 
model of care of IPF patients considering Hambly et al.' study. Table 4 of the manuscript (11) is a brilliant example of the positive effects that the care coordinator may have on the patients. Availability, comfort, confidence, education, efficiency and education and coordination were the themes that the patients identified as plus versus the usual care. All together these items can be grouped under the umbrella of a better "patient-clinician" relationship. Communication between health care professionals and patients/family members is about dialogue, especially when the patient needs to better understand the nature of the disease, the treatment proposed and the outcome, that we know in IPF could be very poor. Each person in the dialogue brings with them their own assumptions, behavior styles, expectations and goals, but unfortunately very often, due to several reasons (i.e., lack of time and lack of expertise and training in dealing with communication by health care professional), the communication between the patient and the clinicians is poor and/or insufficient. It has been shown for example that when an "hurry" physician is talking with a patient and/or with relatives, usually speak for more than $50 \%$ of the total length of the speech, and therefore the satisfaction of the patient and family is low (12-17). Conversely, when we give them more time to ask questions, to express doubts or expectations, the quality of the interaction dramatically improves (12-14). Listening is not a passive act but a very active one. We have found in this respect very interesting what a patient reported [see table 4 of Hambly et al. publication (11)]. "I am a new IPF patient and being able to call someone and get answers to my questions immediately has been a comfort. You don't feel so alone and the thoughtful, caring person on the other end of the phone was always able to allay my fears and answer my questions." The aim of active listening is to elicit the feelings, treatment expectations and various problems experienced in real life by patients, accounting also social aspects and family care. The possibility given in the Hambly et al. study, by the high coordinator use scenario, was likely to build trusting and caring relationship, reducing uncertainty, enhances patients' coping and may be foster realistic hope (11). For example, as previously stated, a subgroup of IPF patients are quite reluctant to undergo an anti-fibrotic treatment, partly because of the complexity of the dosage schedule (i.e., mainly for pirfenidone) and the fear of side effect $(9,10)$. To address patients concerns through picking up cues and listening to their doubts may have improved in this study the understanding of the benefits and also to cope with adverse of anti-fibrotic therapy, if they had occurred.
Indeed, despite this was not clearly pointed out [see ref. 2 and 3 of Hambly's study (11)] or quantified in the paper, the care coordinator was probably in charge also of starting an "early palliative" care program.

Prognosis of IPF is fatal: the mean survival is reported to be 4 years (1-4), and therefore it has been suggested to initiate this approach in the early stage of the disease, not waiting therefore when symptoms become very severe. Indeed, the whole population of the study (11) have got the diagnosis on average 2.5 years before enrolment and half of them were already on long-term oxygen therapy. It has been shown for example that a dedicated care service may improve refractory dyspnea in patients with advanced respiratory diseases, including IPF. Knowing that the disease is fatal, it is very likely that the IPF patient, as demonstrated for COPD patients by Curtis (18) may have asked to the care coordinator "how long do I have to live" and "what dying might be like". Open communication does not destroy hope. The evidence shows that most people want to know the truth and cope better if they are supported through honest discussion about their situation. When done sensitively open communication enhances hope and helps patients to find their strength when dealing with difficult situations, picking up eventually patients' cues and respond and allow patients' concerns to be explored.

Another important finding of the study (11) is that the HRQoL presented not so many differences between HCU and LCU clinics despite in the HCU clinic patients declared an increased satisfaction with this novel model $(\mathrm{P}<0.05)$ compared to the one they received before being enrolled in the HCU group, while LCU patients did not. Nevertheless, in the HCU clinic the care coordinator was estimated to alleviate approximately one-third of the physician's IPFrelated workload, and to facilitate the care of more patients per physician. This also resulted in an annual cost-saving of about $\$ 137,000$. It was not clear from the study how the hours of care per patient were calculated (probably self-reported), but this bias is still not likely to influence the overall results. Saving time for the doctors is a critical issue. It has been demonstrated that physicians reported frustration and concern about the progressive reduction of the face-to-face time they can spend with patients and that their administrative burdens are intensifying. Inadequate time for visits is perceived also badly from a patient's point of view, lowering patient satisfaction and reducing their quality of care (12-17). Having enough time to give exhaustive information to patients, sharing with them treatment and follow-up decisions is a crucial point to 
offer a good-quality care and, simultaneously, to maintain patient's compliance through a relationship of trust with the threating physician. The role of the care coordinator could be inserted in these aspects optimizing the physician's role and competences in these tasks.

The Hambly's study (11) has also some limitations that need to be discussed. First, the L-IPFi questionnaire was used to assess the impact of the coordinator on IPF patient HRQoL; this questionnaire was developed for IPF patients, however the time and the way this was filled by the patients, was not enough specified in the text. We know that the time elapsed in a clinician-patient relationship may have an important effect on the patient's satisfaction, as documented for example in COPD patients. Indeed the major factor in a patient-clinician interview or filling a questionnaire is how it is conducted. It has been reported that some patient "little white lies" in answering physician-administrated questionnaire are quite common; the principal reason for that is the patient attempt to minimize his or her bad experiences to please their treating physician. Despite the study assessed an interesting issue, the small sample size, and the lack of a randomization, makes the results of the investigation somehow difficult to interpret and to be generalized.

Optimizing quality of life in patients affected by IPF is an urgent need in world health care. Even with some limits, the study of Hambly et al. contribute to address this topic and suggests that improving QoL of IPF patients and a teamwork-based management could also represent a costsaving strategy.

\section{Acknowledgments}

We thank Editors for the invitation to write this editorial in such an interesting topic on an urgent issue in IPF management.

Funding: None.

\section{Footnote}

Provenance and Peer Review: This article was commissioned by the editorial office, Fournal of Thoracic Disease. The article did not undergo external peer review.

Conflicts of Interest: Both authors have completed the ICMJE uniform disclosure form (available at http://dx.doi. org/10.21037/jtd.2020.04.44). The authors have no conflicts of interest to declare.
Ethical Statement: The authors are accountable for all aspects of the work in ensuring that questions related to the accuracy or integrity of any part of the work are appropriately investigated and resolved.

Open Access Statement: This is an Open Access article distributed in accordance with the Creative Commons Attribution-NonCommercial-NoDerivs 4.0 International License (CC BY-NC-ND 4.0), which permits the noncommercial replication and distribution of the article with the strict proviso that no changes or edits are made and the original work is properly cited (including links to both the formal publication through the relevant DOI and the license). See: https://creativecommons.org/licenses/by-nc-nd/4.0/.

\section{References}

1. Raghu G, Remy-Jardin M, Myers JL, et al. Diagnosis of Idiopathic Pulmonary Fibrosis. An Official ATS/ERS/JRS/ ALAT Clinical Practice Guideline. Am J Respir Crit Care Med 2018;198:e44-68.

2. Meltzer EB, Noble PW. Idiopathic pulmonary fibrosis. Orphanet J Rare Dis 2008;3:8.

3. Ryerson CJ, Arean PA, Berkeley J, et al. Depression is a common and chronic comorbidity in patients with interstitial lung disease. Respirology 2012;17:525-32.

4. Zou RH, Kass DJ, Gibson KF, et al. The Role of Palliative Care in Reducing Symptoms and Improving Quality of Life for Patients with Idiopathic Pulmonary Fibrosis: A Review. Pulm Ther 2020;6:35-46.

5. Zurkova M, Kriegova E, Kolek V, et al. Effect of pirfenidone on lung function decline and survival: 5 -yr experience from a real-life IPF cohort from the Czech EMPIRE registry. Respir Res 2019;20:16.

6. Flaherty KR, Fell CD, Huggins JT, et al. Safety of nintedanib added to pirfenidone treatment for idiopathic pulmonary fibrosis. Eur Respir J 2018;52:1800230.

7. Rodríguez-Portal JA. Efficacy and Safety of Nintedanib for the Treatment of Idiopathic Pulmonary Fibrosis: An Update. Drugs R D 2018;18:19-25.

8. Matsuda T, Taniguchi H, Ando M, et al. Depression Is Significantly Associated with the Health Status in Patients with Idiopathic Pulmonary Fibrosis. Intern Med 2017;56:1637-44.

9. Maher TM, Swigris JJ, Kreuter M, et al. Identifying Barriers to Idiopathic Pulmonary Fibrosis Treatment: A Survey of Patient and Physician Views. Respiration 
2018;96:514-24.

10. Maher TM, Strek ME. Antifibrotic therapy for idiopathic pulmonary fibrosis: time to treat. Respir Res 2019;20:205.

11. Hambly N, Goodwin S, Aziz-Ur-Rehman A, et al. A cross-sectional evaluation of the idiopathic pulmonary fibrosis patient satisfaction and quality of life with a care coordinator. J Thorac Dis 2019;11:5547-56.

12. Burdi MD, Baker LC. Physicians' perceptions of autonomy and satisfaction in California. Health Aff (Millwood) 1999;18:134-45.

13. Lin CT, Albertson GA, Schilling LM, et al. Is patients' perception of time spent with the physician a determinant of ambulatory patient satisfaction? Arch Intern Med

Cite this article as: Nava S, Prediletto I. Idiopathic pulmonary fibrosis management: caring is sparing. J Thorac Dis 2020;12(10):5265-5268. doi: 10.21037/jtd.2020.04.44
2001;161:1437-42.

14. Gross DA, Zyzanski SJ, Borawski EA, et al. Patient satisfaction with time spent with their physician. J Fam Pract 1998;47:133-7.

15. Mechanic D. Physician discontent: challenges and opportunities. JAMA 2003;290:941-6.

16. Kassirer JP. Doctor discontent. N Engl J Med 1998;339:1543-5.

17. Palmieri JJ, Stern TA. Lies in the doctor-patient relationship. Prim Care Companion J Clin Psychiatry 2009;11:163-8.

18. Curtis JR. Palliative and end-of-life care for patients with severe COPD. Eur Respir J 2008;32:796-803. 